\title{
Systematic Review \\ Dose-Intense Cisplatin-Based Neoadjuvant Chemotherapy Increases Survival in Advanced Cervical Cancer: An Up-to-Date Meta-Analysis
}

\author{
Van Tai Nguyen 1,2,3 (D), Sabine Winterman ${ }^{2}$, Margot Playe ${ }^{4}$, Amélie Benbara ${ }^{5}$, Laurent Zelek ${ }^{2,6}$, \\ Frédéric Pamoukdjian $3,7,+$ and Guilhem Bousquet $2,3,6, *,+(\mathbb{D}$
}

Citation: Nguyen, V.T.; Winterman,

S.; Playe, M.; Benbara, A.; Zelek, L.;

Pamoukdjian, F.; Bousquet, G.

Dose-Intense Cisplatin-Based

Neoadjuvant Chemotherapy

Increases Survival in Advanced

Cervical Cancer: An Up-to-Date

Meta-Analysis. Cancers 2022, 14, 842.

https://doi.org/10.3390/

cancers14030842

Academic Editors: Thomas P.

Condras and George Larry Maxwell

Received: 1 December 2021

Accepted: 1 February 2022

Published: 8 February 2022

Publisher's Note: MDPI stays neutral with regard to jurisdictional claims in published maps and institutional affiliations.

Copyright: () 2022 by the authors. Licensee MDPI, Basel, Switzerland. This article is an open access article distributed under the terms and conditions of the Creative Commons Attribution (CC BY) license (https:/ / creativecommons.org/licenses/by/ $4.0 /)$.
1 National Cancer Hospital, Department of Medical Oncology 1, Hanoi 10000, Vietnam; van-tai.nguyen@inserm.fr

2 Assistance Publique Hôpitaux de Paris, Hôpital Avicenne, Service d'Oncologie Médicale, 93000 Bobigny, France; sabine.winterman@aphp.fr (S.W.); laurent.zelek@aphp.fr (L.Z.)

3 Institut National de la Santé et de la Recherche Médicale (INSERM), UMR_S942, Université de Paris-Université Sorbonne Paris Nord, 93000 Bobigny, France; frederic.pamoukdjian@aphp.fr

4 Assistance Publique Hôpitaux de Paris, Hôpital Avicenne, Service de Médecine Nucléaire, 93000 Bobigny, France; margot.playe@aphp.fr

5 Assistance Publique Hôpitaux de Paris, Hôpital Jean Verdier, Service de Gynécologie-Obstétrique, 93140 Bondy, France; amelie.benbara@aphp.fr

6 Université Sorbonne Paris Nord, 9 Avenue Jean Baptiste Clément, 93439 Villetaneuse, France

7 Assistance Publique Hôpitaux de Paris, Hôpital Avicenne, Service de Médecine Gériatrique, 93000 Bobigny, France

* Correspondence: guilhem.bousquet@aphp.fr

$+\quad$ These authors are co-senior authors.

Simple Summary: Although neoadjuvant chemotherapy has become standard care for many locally advanced cancer sites, the benefit of neoadjuvant chemotherapy remains unclear for the treatment of locally advanced cervical cancer. With this meta-analysis, we set out to demonstrate the benefit of using dose-intense cisplatin-based neoadjuvant chemotherapy in terms of overall survival and progression-free survival. Dose-intense cisplatin-based neoadjuvant chemotherapy followed by local therapy was significantly associated with a survival benefit in the treatment of locally advanced cervical carcinoma. Even though radiotherapy combined with weekly cisplatin-based chemotherapy remains standard of care for the treatment of locally advanced cervical cancer, our meta-analysis makes it possible to consider the use of dose-intense cisplatin-based neoadjuvant chemotherapy when local treatment is suboptimal and opens perspectives for designing new clinical trials in this setting.

Abstract: Purpose: We set out to demonstrate the benefit of using dose-intense cisplatin-based neoadjuvant chemotherapy in terms of overall survival and progression-free survival. Methods: We searched through MEDLINE and Cochrane Library databases up to May 2021 to identify randomized clinical trials comparing the benefit of using cisplatin-based neoadjuvant chemotherapy followed by local treatment with local treatment alone for the treatment of locally advanced cervical cancer. The PRISMA statement was applied. Results: Twenty-two randomized clinical trials were retrieved between 1991 and 2019, corresponding to 3632 women with FIGO stages IB2-IVA cervical cancer. More than $50 \%$ of the randomized clinical trials were assessed as having a low risk of bias. There was no benefit of neoadjuvant chemotherapy on overall survival, but there was significant heterogeneity across studies $\left(\mathrm{I}^{2}=45 \%, p=0.01\right)$. In contrast, dose-intense cisplatin at over $72.5 \mathrm{mg} / \mathrm{m}^{2} / 3$ weeks was significantly associated with increased overall survival $(R R=0.87, p<0.05)$ with no heterogeneity across the pooled studies $\left(\mathrm{I}^{2}=36 \%, p=0.11\right)$. The survival benefit was even greater when cisplatin was administered at a dose over $105 \mathrm{mg} / \mathrm{m}^{2} / 3$ weeks $(R R=0.79, p<0.05)$. Conclusion: Even though radiotherapy combined with weekly cisplatin-based chemotherapy remains standard of care for the treatment of locally advanced cervical cancer, our meta-analysis makes it possible to consider the use of dose-intense cisplatin-based neoadjuvant chemotherapy when local treatment is suboptimal and opens perspectives for designing new clinical trials in this setting. Neoadjuvant chemotherapy 
could be proposed when surgery is local treatment instead of standard chemoradiotherapy for the treatment of locally advanced cervical cancer.

Keywords: locally advanced cervical cancer; neoadjuvant therapy; dose-intense cisplatin-based

\section{Introduction}

Cervical cancer is the fourth most common cancer among women, with an estimated 604,1270 new cases in 2020 worldwide [1]. It is also the fourth cause of death from cancer among women [1], and 5-year overall survival rates range from $75 \%$ to $22 \%$ for locally advanced stages IB2 to IVA according to the International Federation of Gynecology and Obstetrics (FIGO) [2]. For locally advanced stages, the standard treatment is whole pelvic radiotherapy combined with cisplatin-based chemotherapy [3,4]. However, barriers to radiotherapy access is a major issue in low-income countries where the incidence and mortality of cervical cancer is higher than in middle- and upper-income countries [5-7]. This may be also true in rich countries where daily access to radiotherapy can be difficult in the case of long travel distances [8,9]. In these circumstances, surgery as a local treatment is usually proposed to women. Indeed, for the management of women with invasive cervical cancer, the American Society of Clinical Oncology has recommended that "In basic settings where patients cannot be treated with radiation therapy, extrafascial hysterectomy either alone or after chemotherapy may be an option for women with stage IA1 to IVA cervical cancer" [10].

Neoadjuvant chemotherapy followed by local treatment of primary tumors has become standard care for many locally advanced cancer sites [11-13]. However, for cervical cancer, the benefit of neoadjuvant chemotherapy remains controversial, mainly because of the heterogeneous nature of clinical trials, including different clinical stages and various treatment protocols [14]. Cisplatin is the most effective drug for the treatment of cervical cancer, for both locally advanced and metastatic stages $[15,16]$, and resistance to cisplatin is a major cause of relapse and mortality. Cisplatin mainly acts through the formation of platinum-DNA adducts, thus inducing double-strand DNA breaks $[17,18]$. Cisplatin is particularly efficacious in cancers with DNA-repair pathway defects, including ovarian and breast cancers $[19,20]$. In contrast, for cervical cancer, the activation of DNA-repair pathways with overexpression of ERCC1, RAD51, or PARP1 may lead to cisplatin resistance [21-23]. In the neoadjuvant settings, on patient pretreated biopsies, a high level of $\mathrm{HSPB} 1 / \mathrm{p}$ as a molecular marker of DNA damage and repair were associated with a lower response to cisplatin [24]. Higher doses of cisplatin using a dose-intense regimen could be a way to overcome resistance to cisplatin. For metastatic disease, cisplatin-based triplet regimens have improved response rates and survival compared to monotherapy or doublet regimen, but the benefit of using a dose-intense cisplatin-based regimen has not yet been demonstrated [16].

In this meta-analysis, we intended to demonstrate the benefit of using dose-intense cisplatin-based neoadjuvant chemotherapy for the treatment of locally advanced cervical cancer and see if neoadjuvant chemotherapy could be more systematically proposed when surgery is the local treatment instead of standard chemoradiotherapy.

\section{Materials and Methods}

\subsection{Search Strategy and Selection Criteria}

The Preferred Reporting Items for Systematic reviews and Meta-Analyses (PRISMA) method was applied for this meta-analysis [25]. For the search strategy, we applied the following method: using an ad hoc algorithm composed of both thesaurus and free-text terms, we searched MEDLINE via PubMed and Cochrane Library for articles published up to May 2021. In addition, the references contained in the articles and relevant reviews identified were also considered to avoid eligible articles being missed. The algorithm 
was the following: ("Uterine Cervical Neoplasms" [MeSH] OR "Cervix Neoplasms" OR "Cervix Cancer" OR "Cervical Neoplasm") AND ("Locally Advanced") AND ("Neoadjuvant Therapy"[MeSH] OR “Neoadjuvant Chemotherapy"). The International Prospective Register of Systematic Review (PROSPERO) study's Registration Number CRD42021245170 in April 2021.

For study selection, we applied the Population, Intervention, Comparator group, Outcomes, and Study design (PICOS) criteria [25] as detailed in Supplementary Table S1. The studies that were included in this meta-analysis met the following inclusion criteria: (i) all studies were randomized clinical trials; (ii) the patients had stages IB2 to IVA cervical cancer; (iii) the patients received or did not receive cisplatin-based neoadjuvant chemotherapy followed by local treatment including chemoradiotherapy or radiotherapy alone or surgery alone; (iv) the study endpoints were overall survival and progression-free survival. Overall survival (OS) was defined as the time interval between the date of randomization and the date of death from any cause or the last follow-up. Progression-free survival (PFS) was defined as the time from randomization until the first confirmation of progression or death from any cause.

Two authors (V.T.N. and G.B.) independently screened the papers retrieved, initially by titles, then by abstracts, and finally by full texts. In the case of discordance, a third author (F.P.) was solicited to make the decision whether or not to retrieve the paper. We identified a total of 22 relevant publications. The quality of the randomized clinical trials was evaluated using the Cochrane Handbook for Systematic Reviews of Interventions (CHSRI) guidelines, and the risk of bias was classified as low, unclear, or high [26].

\subsection{Statistical Analyses}

The data were analyzed using $\mathrm{R}$ statistical software (version 4.0.3; R Foundation for Statistical Computing, Vienna, Austria; http: / / www.r-project.org, accessed on 27 March 2021). Categorical variables were summarized as numbers (percentage), and continuous variables were summarized as means \pm standard deviation (SD) or medians \pm interquartile range (IQR). On the articles selected, we performed a meta-analysis (with the package "meta") to assess the prognostic value of neoadjuvant cisplatin-based chemotherapy in locally advanced cervical cancers for overall survival (OS) and progression-free survival (PFS). We assessed the heterogeneity of study results by using the $\mathrm{I}^{2}$ indicator and Cochran's $Q$ test. $\mathrm{I}^{2}$ values of $0 \%, 25 \%, 50 \%$, and $75 \%$ were considered to indicate absence of heterogeneity and low, moderate, and high heterogeneity, respectively. A value of $p \leq 0.05$ on the $\mathrm{Q}$ test indicated significant heterogeneity. Graphically, the pooled results were summarized as risk ratios (RRs) and their $95 \%$ confidence interval $(95 \% \mathrm{CI})$ in a forest plot using fixed or random effect as appropriate. Publication bias was assessed both graphically using a funnel plot and quantitatively with a linear regression test of funnel plot asymmetry (nonsignificant $p$ value meaning no publication bias). All tests were two sided, and the threshold for statistical significance was set at a value of $p<0.05$.

\section{Results}

The literature search and screening processes are detailed in Figure 1. The search algorithm initially identified 426 articles, and 12 additional articles were considered after reading the references or related reviews. After careful screening, 416 articles were excluded, most of them being nonrandomized trials (74) or trials with a nonrelevant outcome (108). The articles excluded at the full-text screening stage are listed in Supplementary Table S2. Twenty-two articles were finally retrieved for this meta-analysis. 


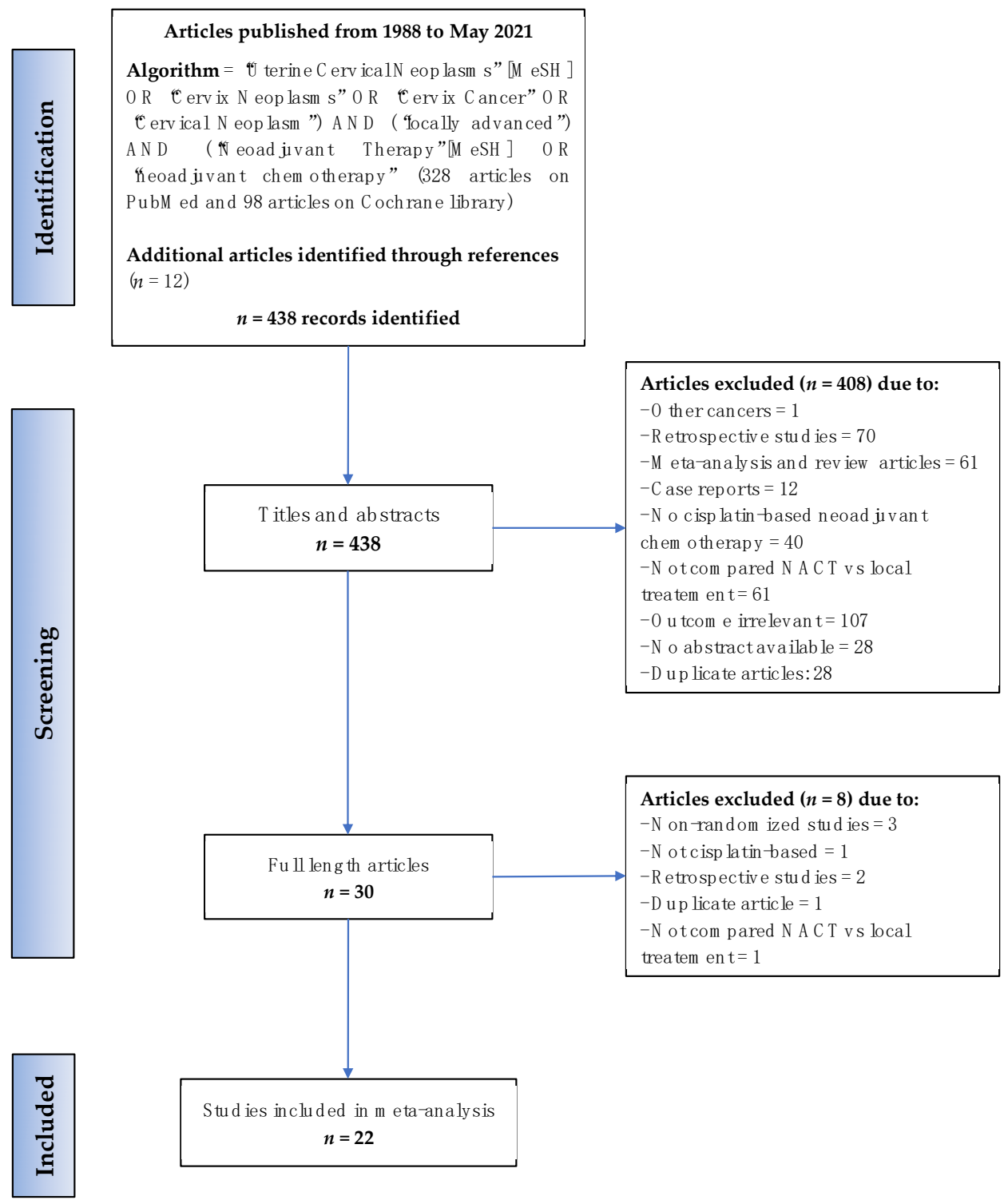

Figure 1. Flow diagram of the search strategy. MeSH, Medical Subject Headings; NACT, neoadjuvant chemotherapy.

These 22 eligible randomized studies (14 phase II and 8 phase III clinical trials) included a total of 3632 women with stages IB2 to IVA cervical cancers. The study characteristics are listed in Table 1. Mean/median ages ranged from 39 to 57 years in the neoadjuvant chemotherapy arm, and the median follow-up time ranged from 16 to 108 months. Briefly, the local treatment was chemoradiotherapy in one study, radiotherapy in fourteen studies, and surgery in seven studies. We found that the three studies by Sardi et al. [27-29], and the two studies by Tattersall et al. [30,31], were not overlapping and thus did not concern the same patients included in these five clinical trials conducted by two different teams. Concerning patients treated with neoadjuvant chemotherapy, all received a cisplatin-based regimen with a planned total dose of cisplatin ranging from 100 to $300 \mathrm{mg} / \mathrm{m}^{2}$ over a period ranging from 28 to 63 days. 
Table 1. The main characteristics of the 22 studies included in the meta-analysis.

\begin{tabular}{|c|c|c|c|c|c|c|c|c|c|c|c|}
\hline \multirow{2}{*}{$\begin{array}{c}\text { Country } \\
\text { Author } \\
\text { Year } \\
\text { Trial }\end{array}$} & \multirow{2}{*}{$\begin{array}{l}\text { Median } \\
\text { Follow- } \\
\text { up } \\
\text { (Months) }\end{array}$} & \multirow[t]{2}{*}{ Comparison } & \multicolumn{2}{|c|}{$\begin{array}{c}\text { Experimental } \\
{ }^{*} \text { Arm }\end{array}$} & \multicolumn{2}{|c|}{$\begin{array}{l}\text { Control } \\
\text { Arm ** }\end{array}$} & \multirow[t]{2}{*}{ Figostage } & \multirow{2}{*}{$\begin{array}{l}\text { NACT } \\
\text { Regimen }\end{array}$} & \multirow{2}{*}{$\begin{array}{l}\text { Planned } \\
\text { Duration } \\
\text { of NACT }\end{array}$} & \multirow{2}{*}{$\begin{array}{c}\text { Cisplatin } \\
\left(\mathrm{mg} / \mathrm{m}^{2} / 3\right. \\
\text { Weeks) }\end{array}$} & \multirow[t]{2}{*}{ Radiotherapy } \\
\hline & & & $\mathbf{n}$ & Age ${ }^{\#}$ & $\mathrm{n}$ & Age \# & & & & & \\
\hline $\begin{array}{c}\text { Brazil } \\
\text { da Costa } \\
{[32]} \\
2019 \\
\text { Phase II }\end{array}$ & 31.7 & $\begin{array}{c}\text { NACT+CRT } \\
\text { vs. } \\
\text { CRT }\end{array}$ & 55 & $\begin{array}{c}48 \\
(22-69)\end{array}$ & 52 & $\begin{array}{c}45 \\
(20-67)\end{array}$ & $\begin{array}{c}\text { IIB-IVA } \\
\text { a }\end{array}$ & $\begin{array}{c}\text { CCDP } \\
50 \mathrm{mg} / \mathrm{m}^{2} \\
\mathrm{Gem} \\
1000 \mathrm{mg} / \mathrm{m}^{2} \\
\text { Day } 1,8\end{array}$ & $\begin{array}{c}\text { Every } \\
21 \text { days for } \\
3 \text { cycles }\end{array}$ & 50 & $\begin{array}{c}\text { Weekly } \\
\text { CCDP } \\
40 \mathrm{mg} / \mathrm{m}^{2} \\
\text { with RT } \\
45-50.4 \text { Gy } \\
\text { over } 6 \text { weeks } \\
\text { followed by } \\
\text { BT } 28-30 \text { Gy }\end{array}$ \\
\hline $\begin{array}{c}\text { China } \\
\text { Yang [33] } \\
2016 \\
\text { Phase III }\end{array}$ & 32 & $\begin{array}{c}\text { NACT+surgery } \\
\text { vs. } \\
\text { surgery }\end{array}$ & 109 & $\begin{array}{c}47 \\
(23-66)\end{array}$ & 110 & $\begin{array}{c}48 \\
(26-68)\end{array}$ & IB2-II ${ }^{a}$ & $\begin{array}{c}\text { CCDP } \\
70 \mathrm{mg} / \mathrm{m}^{2} \\
\text { IRI } \\
180 \mathrm{mg} / \mathrm{m}^{2} \\
\text { OR } \\
\text { CCDP } 70 \\
\mathrm{Pacl} \\
175 \mathrm{mg} / \mathrm{m}^{2}\end{array}$ & $\begin{array}{c}\text { Every } \\
21 \text { days for } \\
1-2 \text { cycles }\end{array}$ & 70 & $\begin{array}{c}\text { Postoperative } \\
\text { RT of } \\
48-50 \text { Gy if } \\
\text { risk factors. }\end{array}$ \\
\hline $\begin{array}{c}\text { Japan } \\
\text { Katsumata } \\
{[34]} \\
2013 \\
\text { Phase III }\end{array}$ & 49 & $\begin{array}{c}\text { NACT+surgery } \\
\text { vs. } \\
\text { surgery }\end{array}$ & 67 & $\begin{array}{c}47 \\
(28-70)\end{array}$ & 67 & $\begin{array}{c}46 \\
(22-67)\end{array}$ & $\begin{array}{c}\text { IB2-IIB } \\
\text { b }\end{array}$ & $\begin{array}{c}\text { CCDP } \\
70 \mathrm{mg} / \mathrm{m}^{2} \\
\mathrm{BLM} \\
35 \mathrm{mg} / \mathrm{m}^{2} \\
\mathrm{VCR} \\
0.7 \mathrm{mg} / \mathrm{m}^{2} \\
\mathrm{MMC} \\
7 \mathrm{mg} / \mathrm{m}^{2}\end{array}$ & $\begin{array}{l}\text { Every } \\
21 \text { days for } \\
2 \text { cycles }\end{array}$ & 70 & $\begin{array}{l}\text { Postoperative } \\
\text { RT of } \\
45-50.4 \text { Gy if } \\
\text { risk factors. } \\
\text { BT if surgical } \\
\text { margins } \\
\text { positive }\end{array}$ \\
\hline $\begin{array}{c}\text { Italy } \\
\text { Mossa [35] } \\
2010 \\
\text { Phase III }\end{array}$ & 84 & $\begin{array}{c}\text { NACT+surgery/ } \\
\text { RT vs. } \\
\text { surgery/RT }\end{array}$ & 159 & $\begin{array}{c}48.5 \\
(32-65)\end{array}$ & 129 & $\begin{array}{c}48.5 \\
(32-65)\end{array}$ & $\underset{b}{\text { IB-IIIB }}$ & $\begin{array}{c}\mathrm{CCDP} \\
50 \mathrm{mg} / \mathrm{m}^{2} \\
\mathrm{VCR} \\
1 \mathrm{mg} / \mathrm{m}^{2} \\
\mathrm{BLM} \\
75 \mathrm{mg} / \mathrm{m}^{2}\end{array}$ & $\begin{array}{c}\text { Every } \\
21 \text { days for } \\
3 \text { cycles }\end{array}$ & 50 & $\begin{array}{l}\text { 50 Gy over } \\
5-6 \text { weeks } \\
\text { followed by } \\
\text { BT a } \\
\text { maximum } \\
30 \text { Gy }\end{array}$ \\
\hline $\begin{array}{c}\text { China } \\
\text { Chen [36] } \\
2008 \\
\text { Phase II }\end{array}$ & NA & $\begin{array}{c}\text { NACT+surgery } \\
\text { vs. } \\
\text { surgery }\end{array}$ & 72 & $\begin{array}{c}44 \\
(25-74)\end{array}$ & 70 & $\begin{array}{c}44 \\
(25-74)\end{array}$ & $\begin{array}{c}\text { IB2-IIB } \\
\text { b }\end{array}$ & $\begin{array}{c}\mathrm{CCDP} \\
100 \mathrm{mg} / \mathrm{m}^{2} \\
\mathrm{MMC} \\
20 \mathrm{mg} / \mathrm{m}^{2} \\
5 \mathrm{FU} \\
120 \mathrm{mg} / \mathrm{kg}\end{array}$ & $\begin{array}{c}\text { Every } \\
14 \text { days for } \\
2-3 \text { cycles }\end{array}$ & 150 & $\begin{array}{l}\text { Postoperative } \\
\text { pelvic RT at } \\
\text { a dose of } \\
45 \text { Gy if risk } \\
\text { factors after } \\
\text { surgery }\end{array}$ \\
\hline $\begin{array}{c}\text { American } \\
\text { Eddy [37] } \\
2007 \\
\text { Phase III }\end{array}$ & 62 & $\begin{array}{c}\text { NACT+surgery } \\
\text { vs. } \\
\text { surgery }\end{array}$ & 145 & NA & 143 & NA & $\mathrm{IB} 2^{\mathrm{b}}$ & $\begin{array}{c}\mathrm{CCDP} \\
50 \mathrm{mg} / \mathrm{m}^{2} \\
\mathrm{VCR} \\
1 \mathrm{mg} / \mathrm{m}^{2}\end{array}$ & $\begin{array}{c}\text { Every } \\
10 \text { days for } \\
3 \text { cycles }\end{array}$ & 105 & $\begin{array}{l}\text { Postoperative } \\
\text { pelvic RT if } \\
\text { risk factors } \\
\text { after surgery }\end{array}$ \\
\hline $\begin{array}{c}\text { China } \\
\text { Cai [38] } \\
2006 \\
\text { Phase II }\end{array}$ & 62 & $\begin{array}{c}\text { NACT+surgery } \\
\text { vs. } \\
\text { surgery }\end{array}$ & 52 & 45.6 & 54 & 44.8 & $\mathrm{IB}^{\mathrm{b}}$ & $\begin{array}{c}\text { CCDP } \\
75 \mathrm{mg} / \mathrm{m}^{2} \\
5 \mathrm{FU} \\
120 \mathrm{mg} / \mathrm{kg}\end{array}$ & $\begin{array}{l}\text { Every } \\
21 \text { days for } \\
2 \text { cycles }\end{array}$ & 75 & $\begin{array}{l}\text { Postoperative } \\
\text { RT at a dose } \\
\text { of } 45 \text { Gy if } \\
\text { risk factors } \\
\text { after surgery }\end{array}$ \\
\hline $\begin{array}{c}\text { Italy } \\
\text { Tabata [39] } \\
2003 \\
\text { Phase II }\end{array}$ & NA & $\begin{array}{c}\text { NACT+RT } \\
\text { vs. } \\
\text { RT }\end{array}$ & 32 & $\begin{array}{c}57 \\
(35-68)\end{array}$ & 29 & $\begin{array}{c}59 \\
(44-70)\end{array}$ & $\begin{array}{l}\text { IIIB- } \\
\text { IVA } \\
\mathrm{b}\end{array}$ & $\begin{array}{c}\mathrm{CCDP} \\
70 \mathrm{mg} / \mathrm{m}^{2} \\
\mathrm{BLM} \\
5 \mathrm{mg} / \mathrm{m}^{2} \\
\mathrm{VCR} \\
0.7 \mathrm{mg} / \mathrm{m}^{2} \\
\mathrm{MMC} \\
7 \mathrm{mg} / \mathrm{m}^{2}\end{array}$ & $\begin{array}{c}\text { Every } \\
28 \text { days for } \\
3 \text { cycles }\end{array}$ & 52.5 & $\begin{array}{c}50 \text { Gy in } 25 \mathrm{~F} \\
\text { followed by } \\
\text { BT }\end{array}$ \\
\hline $\begin{array}{c}\text { Italy } \\
\text { Benedetti- } \\
\text { Panici [40] } \\
2002 \\
\text { Phase III }\end{array}$ & 40 & $\begin{array}{c}\text { NACT+surgery } \\
\text { vs. } \\
\text { RT }\end{array}$ & 227 & $\begin{array}{c}49 \\
(25-70)\end{array}$ & 214 & $\begin{array}{c}52 \\
(28-69)\end{array}$ & IB2-III ${ }^{b}$ & $\begin{array}{c}\mathrm{CCDP} \\
160 \mathrm{mg} / \mathrm{m}^{2} \\
\mathrm{BLM} \\
15 \mathrm{mg} / \mathrm{m}^{2}, \\
\text { Day } 1,8\end{array}$ & $\begin{array}{l}\text { Every } \\
21 \text { days for } \\
2 \text { cycles }\end{array}$ & $160 \S$ & $\begin{array}{l}\text { Median total } \\
\text { dose of } \\
70 \text { Gy } \\
\text { delivered to } \\
\text { point A over } \\
62 \text { days }\end{array}$ \\
\hline
\end{tabular}


Table 1. Cont.

\begin{tabular}{|c|c|c|c|c|c|c|c|c|c|c|c|}
\hline \multirow{2}{*}{$\begin{array}{l}\text { Country } \\
\text { Author } \\
\text { Year } \\
\text { Trial }\end{array}$} & \multirow{2}{*}{$\begin{array}{l}\text { Median } \\
\text { Follow- } \\
\text { up } \\
\text { (Months) }\end{array}$} & \multirow{2}{*}{ Comparison } & \multicolumn{2}{|c|}{$\begin{array}{c}\text { Experimental } \\
* \text { Arm }\end{array}$} & \multicolumn{2}{|c|}{$\begin{array}{l}\text { Control } \\
\text { Arm ** }\end{array}$} & \multirow{2}{*}{ Figostage } & \multirow{2}{*}{$\begin{array}{c}\text { NACT } \\
\text { Regimen }\end{array}$} & \multirow{2}{*}{$\begin{array}{l}\text { Planned } \\
\text { Duration } \\
\text { of NACT }\end{array}$} & \multirow{2}{*}{$\begin{array}{c}\text { Cisplatin } \\
\left(\mathrm{mg} / \mathrm{m}^{2} / 3\right. \\
\text { Weeks })\end{array}$} & \multirow{2}{*}{ Radiotherapy } \\
\hline & & & $\mathbf{n}$ & Age ${ }^{\#}$ & $\mathrm{n}$ & Age ${ }^{\#}$ & & & & & \\
\hline $\begin{array}{c}\text { China } \\
\text { Chang [41] } \\
2000 \\
\text { Phase II }\end{array}$ & 39 & $\begin{array}{c}\text { NACT+surgery } \\
\text { vs. } \\
\text { RT }\end{array}$ & 68 & $\begin{array}{c}46 \\
(33-69)\end{array}$ & 52 & $\begin{array}{c}47 \\
(32-70)\end{array}$ & $\begin{array}{l}\text { IB2-IIA } \\
\mathrm{b}\end{array}$ & $\begin{array}{c}\text { CCDP } \\
50 \mathrm{mg} / \mathrm{m}^{2} \\
\mathrm{VCR} \\
1 \mathrm{mg} / \mathrm{m}^{2} \\
\mathrm{BLM} \\
75 \mathrm{mg} / \mathrm{m}^{2}\end{array}$ & $\begin{array}{c}\text { Every } 10 \\
\text { days for } 3 \\
\text { cycles }\end{array}$ & 105 & $\begin{array}{l}\text { 50-54 Gy } \\
\text { followed by } \\
\text { BT, or } 70 \text { Gy } \\
\text { without BT }\end{array}$ \\
\hline $\begin{array}{c}\text { England } \\
\text { Herod [42] } \\
2000 \\
\text { Phase III }\end{array}$ & 108 & $\begin{array}{c}\text { NACT+RT } \\
\text { vs. } \\
\text { RT }\end{array}$ & 86 & $\begin{array}{c}47 \\
(24-74)\end{array}$ & 86 & $\begin{array}{c}46 \\
(27-73)\end{array}$ & $\underset{\mathrm{b}}{\text { IB-IVA }}$ & $\begin{array}{c}\text { CCDP } \\
50 \mathrm{mg} / \mathrm{m}^{2} \\
\text { BLM } 30 \mathrm{mg} \\
\text { IFOS } 5 \mathrm{~g} / \mathrm{m}^{2} \\
\text { Mesna } \\
6 \mathrm{~g} / \mathrm{m}^{2}\end{array}$ & $\begin{array}{c}\text { Every } \\
21 \text { days for } \\
2-3 \text { cycles }\end{array}$ & 50 & $\begin{array}{l}\text { According to } \\
\text { institutional } \\
\text { policy }\end{array}$ \\
\hline $\begin{array}{c}\text { England } \\
\text { Symonds } \\
\text { [43] } \\
2000 \\
\text { Phase III }\end{array}$ & 65 & $\begin{array}{c}\text { NACT+RT } \\
\text { vs. } \\
\text { RT }\end{array}$ & 100 & $\begin{array}{c}49 \\
(25-69)\end{array}$ & 104 & $\begin{array}{c}48 \\
(24-70)\end{array}$ & $\underset{\mathrm{b}}{\mathrm{IIB}-\mathrm{IVA}}$ & $\begin{array}{c}\text { CCDP } \\
50 \mathrm{mg} / \mathrm{m}^{2} \\
\mathrm{MTX} \\
100 \mathrm{mg} / \mathrm{m}^{2}\end{array}$ & $\begin{array}{c}\text { Every } \\
14 \text { days for } \\
3 \text { cycles }\end{array}$ & 75 & $\begin{array}{c}\text { 40-45 Gy in } \\
20 \text { F over } \\
28 \text { days } \\
\text { followed by } \\
\text { BT } \\
24-33.75 \text { Gy }\end{array}$ \\
\hline $\begin{array}{c}\text { Argentina } \\
\text { Sardi [29] } \\
1998 \\
\text { Phase II }\end{array}$ & 84 & $\begin{array}{c}\text { NACT+RT } \\
\text { vs. } \\
\text { RT }\end{array}$ & 73 & 42.9 & 74 & 41.5 & $\mathrm{IIB}^{\mathrm{c}}$ & $\begin{array}{c}\text { CCDP } \\
50 \mathrm{mg} / \mathrm{m}^{2} \\
\mathrm{VCR} \\
1 \mathrm{mg} / \mathrm{m}^{2} \\
\mathrm{BLM} \\
75 \mathrm{mg} / \mathrm{m}^{2}\end{array}$ & $\begin{array}{c}\text { Every } \\
10 \text { days for } \\
3 \text { cycles }\end{array}$ & 105 & $\begin{array}{l}50-60 \text { Gy in } \\
28-30 \text { F over } \\
45-50 \text { days, } \\
\text { followed by } \\
\text { BT } 25-35 \text { Gy }\end{array}$ \\
\hline $\begin{array}{c}\text { India } \\
\text { Kumar } \\
\text { [44] } \\
1998 \\
\text { Phase II }\end{array}$ & NA & $\begin{array}{c}\text { NACT+RT } \\
\text { vs. } \\
\text { RT }\end{array}$ & 88 & $\begin{array}{c}45 \\
(30-65)\end{array}$ & 85 & $\begin{array}{c}45.5 \\
(21-65)\end{array}$ & $\underset{c}{\text { IIB-IVA }}$ & $\begin{array}{c}\text { CCDP } \\
50 \mathrm{mg} / \mathrm{m}^{2} \\
\text { IFOS } 5 \mathrm{~g} / \mathrm{m}^{2} \\
\mathrm{BLM} \\
15 \mathrm{mg} / \mathrm{m}^{2} \\
\mathrm{Mesna} \\
3 \mathrm{~g} / \mathrm{m}^{2}\end{array}$ & $\begin{array}{c}\text { Every } \\
21 \text { days for } \\
2 \text { cycles }\end{array}$ & 50 & $\begin{array}{c}40 \text { Gy in } \\
22 \mathrm{~F}+10 \mathrm{~Gy} \\
\text { in } 5 \text { F over } \\
35 \text { days } \\
\text { followed by } \\
\text { BT } 30 \text { Gy }\end{array}$ \\
\hline $\begin{array}{c}\text { Argentina } \\
\text { Sardi [28] } \\
1997 \\
\text { Phase II }\end{array}$ & 67 & $\begin{array}{c}\text { NACT }+ \\
\text { surgery } \pm \text { RT } \\
\text { vs. } \\
\text { Surgery } \pm \text { RT }\end{array}$ & 102 & $\begin{array}{c}39 \\
(23-68)\end{array}$ & 103 & $\begin{array}{c}41 \\
(24-69)\end{array}$ & $\mathrm{IB}^{\mathrm{c}}$ & $\begin{array}{c}\text { CCDP } \\
50 \mathrm{mg} / \mathrm{m}^{2} \\
\mathrm{VCR} \\
1 \mathrm{mg} / \mathrm{m}^{2} \\
\mathrm{BLM} \\
75 \mathrm{mg} / \mathrm{m}^{2}\end{array}$ & $\begin{array}{c}\text { Every } \\
10 \text { days for } \\
3 \text { cycles }\end{array}$ & 105 & $\begin{array}{c}\text { 50-60 Gy } \\
\text { over } \\
45-50 \text { days } \\
\text { followed by } \\
\text { BT } 25-35 \text { Gy }\end{array}$ \\
\hline $\begin{array}{c}\text { Argentina } \\
\text { Sardi [27] } \\
1996 \\
\text { Phase II }\end{array}$ & 28 & $\begin{array}{c}\text { NACT+RT } \\
\text { vs. } \\
\text { RT }\end{array}$ & 54 & 48.2 & 54 & 49.6 & IIIB $^{c}$ & $\begin{array}{c}\text { CCDP } \\
50 \mathrm{mg} / \mathrm{m}^{2} \\
\mathrm{VCR} \\
1 \mathrm{mg} / \mathrm{m}^{2} \\
\mathrm{BLM} \\
75 \mathrm{mg} / \mathrm{m}^{2}\end{array}$ & $\begin{array}{c}\text { Every } \\
10 \text { days for } \\
3 \text { cycles }\end{array}$ & 105 & $\begin{array}{c}\text { 50-60 Gy } \\
\text { over } \\
45-50 \text { days } \\
\text { followed by } \\
\text { BT } 25-35 \text { Gy }\end{array}$ \\
\hline $\begin{array}{c}\text { Norway } \\
\text { Sundfor } \\
\text { [45] } \\
1996 \\
\text { Phase II }\end{array}$ & 46 & $\begin{array}{c}\text { NACT+RT } \\
\text { vs. } \\
\text { RT }\end{array}$ & 47 & $\begin{array}{c}52.7 \\
(25-70)\end{array}$ & 47 & $\begin{array}{c}52.2 \\
(26-70)\end{array}$ & $\begin{array}{l}\text { IIIB- } \\
\text { IVA } \\
\mathrm{c}\end{array}$ & $\begin{array}{c}\text { CCDP } \\
100 \mathrm{mg} / \mathrm{m}^{2} \\
5 \mathrm{FU} \\
5000 \mathrm{mg} / \mathrm{m}^{2}\end{array}$ & $\begin{array}{c}\text { Every } \\
21 \text { days for } \\
3 \text { cycles }\end{array}$ & 100 & $\begin{array}{c}64.8 \text { Gy in } \\
36 \text { F over } \\
50 \text { days }\end{array}$ \\
\hline $\begin{array}{c}\text { Japan } \\
\text { Kigawa } \\
\text { [46] } \\
1996 \\
\text { Phase II }\end{array}$ & 42 & $\begin{array}{c}\text { NACT } \\
\& \pm \text { surgery } \\
\pm \text { RT } \\
\text { vs. } \\
\text { RT }\end{array}$ & 25 & $\begin{array}{c}55.6 \\
(41-67)\end{array}$ & 25 & $\begin{array}{c}60.2 \\
(43-69)\end{array}$ & $\underset{c}{\text { IIB-IIIB }}$ & $\begin{array}{c}\text { CCDP } \\
50 \mathrm{mg} / \mathrm{m}^{2} \\
\mathrm{BLM} \\
30 \mathrm{mg} / \mathrm{m}^{2}\end{array}$ & $\begin{array}{c}\text { Every } \\
21 \text { days for } \\
2-3 \text { cycles }\end{array}$ & 50 & $\begin{array}{l}50 \text { Gy in } 25 \mathrm{~F} \\
\text { over } 35 \text { days } \\
\text { followed by } \\
\text { BT } 24-38 \text { Gy }\end{array}$ \\
\hline $\begin{array}{c}\text { Australia } \\
\text { Tattersall } \\
\text { [30] } \\
1995 \\
\text { Phase II }\end{array}$ & 16 & $\begin{array}{c}\text { NACT+RT } \\
\text { vs. } \\
\text { RT }\end{array}$ & 129 & $\begin{array}{c}47 \\
(26-75)\end{array}$ & 131 & $\begin{array}{c}52 \\
(27-78)\end{array}$ & $\underset{c}{\text { IIB-IVA }}$ & $\begin{array}{c}\text { CCDP } \\
60 \mathrm{mg} / \mathrm{m}^{2} \\
\mathrm{EPI} \\
110 \mathrm{mg} / \mathrm{m}^{2}\end{array}$ & $\begin{array}{c}\text { Every } \\
21 \text { days for } \\
2-3 \text { cycles }\end{array}$ & 60 & $\begin{array}{c}\text { 40-55 Gy } \\
\text { over } \\
\text { 28-35 days } \\
\text { followed by } \\
\text { BT 30-35 Gy }\end{array}$ \\
\hline
\end{tabular}


Table 1. Cont.

\begin{tabular}{|c|c|c|c|c|c|c|c|c|c|c|c|}
\hline \multirow{2}{*}{$\begin{array}{l}\text { Country } \\
\text { Author } \\
\text { Year } \\
\text { Trial }\end{array}$} & \multirow{2}{*}{$\begin{array}{l}\text { Median } \\
\text { Follow- } \\
\text { up } \\
\text { (Months) }\end{array}$} & \multirow[t]{2}{*}{ Comparison } & \multicolumn{2}{|c|}{$\begin{array}{c}\text { Experimental } \\
* \text { Arm }\end{array}$} & \multicolumn{2}{|c|}{$\begin{array}{l}\text { Control } \\
\text { Arm ** }\end{array}$} & \multirow[t]{2}{*}{ Figostage } & \multirow{2}{*}{$\begin{array}{c}\text { NACT } \\
\text { Regimen }\end{array}$} & \multirow{2}{*}{$\begin{array}{l}\text { Planned } \\
\text { Duration } \\
\text { of NACT }\end{array}$} & \multirow{2}{*}{$\begin{array}{c}\text { Cisplatin } \\
\left(\mathrm{mg} / \mathrm{m}^{2} / 3\right. \\
\text { Weeks })\end{array}$} & \multirow[t]{2}{*}{ Radiotherapy } \\
\hline & & & $\mathbf{n}$ & Age ${ }^{\#}$ & $\mathbf{n}$ & Age ${ }^{\#}$ & & & & & \\
\hline $\begin{array}{c}\text { France } \\
\text { Chauvergne } \\
\text { [47] } \\
1993 \\
\text { Phase III }\end{array}$ & 84 & $\begin{array}{c}\text { NACT+RT } \\
\text { vs. } \\
\text { RT }\end{array}$ & 75 & 54.3 & 76 & 54 & $\underset{c}{\text { IIB-IIIB }}$ & $\begin{array}{c}\text { CCDP } \\
80 \mathrm{mg} / \mathrm{m}^{2} \\
\mathrm{CLB} \\
20 \mathrm{mg} / \mathrm{m}^{2} \\
\mathrm{VCR} \\
0.7 \mathrm{mg} / \mathrm{m}^{2} \\
\mathrm{MTX} \\
30 \mathrm{mg} / \mathrm{m}^{2}\end{array}$ & $\begin{array}{c}\text { Every } \\
21 \text { days for } \\
2-4 \text { cycles }\end{array}$ & 80 & $\begin{array}{c}45 \mathrm{~Gy} \\
\text { followed by } \\
\text { BT }\end{array}$ \\
\hline $\begin{array}{c}\text { Australia } \\
\text { Tattersall } \\
1992[31] \\
\text { Phase II }\end{array}$ & 37 & $\begin{array}{c}\text { NACT+RT } \\
\text { vs. } \\
\text { RT }\end{array}$ & 34 & $\begin{array}{c}54 \\
(33-70)\end{array}$ & 37 & $\begin{array}{c}56 \\
(23-70)\end{array}$ & $\underset{c}{\text { IIB-IVA }}$ & $\begin{array}{c}\text { CCDP } \\
50 \mathrm{mg} / \mathrm{m}^{2} \\
\mathrm{VBL} \\
4 \mathrm{mg} / \mathrm{m}^{2} \\
\mathrm{BLM} \\
45 \mathrm{mg} / \mathrm{m}^{2}\end{array}$ & $\begin{array}{c}\text { Every } \\
21 \text { days for } \\
3 \text { cycles }\end{array}$ & 50 & $\begin{array}{l}40-55 \text { Gy in } \\
20-25 \text { F over } \\
28-35 \text { days }\end{array}$ \\
\hline $\begin{array}{c}\text { Brazil } \\
\text { Souhami } \\
\text { [48] } \\
1991 \\
\text { Phase II }\end{array}$ & 44 & $\begin{array}{c}\text { NACT+RT } \\
\text { vs. } \\
\text { RT }\end{array}$ & 39 & $\begin{array}{c}50 \\
(24-69)\end{array}$ & 52 & $\begin{array}{c}49 \\
(26-69)\end{array}$ & IIIB $^{c}$ & $\begin{array}{c}\text { CCDP } \\
50 \mathrm{mg} / \mathrm{m}^{2} \\
\mathrm{VCR} \\
1 \mathrm{mg} / \mathrm{m}^{2} \\
\mathrm{BLM} 120 \mathrm{U} \\
\mathrm{MMC} \\
10 \mathrm{mg} / \mathrm{m}^{2}\end{array}$ & $\begin{array}{c}\text { Every } 21 \\
\text { days for } 3 \\
\text { cycles }\end{array}$ & 50 & $\begin{array}{c}50 \text { Gy in } 25 \mathrm{~F} \\
\text { over } 35 \text { days } \\
\text { followed by } \\
\text { BT } 40 \mathrm{~Gy}\end{array}$ \\
\hline
\end{tabular}

Abbreviations: NACT, neoadjuvant chemotherapy; RT, radiotherapy; CRT, chemoradiotherapy; FIGO, International Federation of Gynecology and Obstetrics; CDDP, cisplatin; Gem, gemcitabine; IRI, irinotecan; Pacl, paclitaxel; VCR, vincristine; BLM, bleomycin; VBL, vinblastine; IFOS, ifosfamide; 5-FU, 5-fluorouracil; MMC, mitomycin; CLB, chlorambucil; EPI, epirubicin; F, fractions; BT, brachytherapy. NA, not available. ${ }^{\#}$ Age values are presented as median or mean age with minimum and maximum values. ${ }^{*}$ Experimental arm: neoadjuvant chemotherapy arm. ${ }^{* *}$ Control arm: local treatment arm. \& Neoadjuvant intra-arterial infusion chemotherapy. $\S$ The trial used 4 different cisplatin-based regimens with a median cisplatin total dose of $300 \mathrm{mg} / \mathrm{m}^{2}$ over median 39 days. ${ }^{a}$ FIGO staging 2009. ${ }^{b}$ FIGO staging 1994. ${ }^{c}$ FIGO staging 1988 . None of the studies included in the meta-analysis have used the last 2018 FIGO staging [49], and the previous classifications were versions of 1988, 1994, and 2009 FIGO staging.

Regarding progression-free survival, only eighteen of the studies included reported these data, with 1211 events. There was no significant heterogeneity in the overall analysis $\left(\mathrm{I}^{2}=28 \%, p=0.13\right)$, and cisplatin-based neoadjuvant chemotherapy was significantly associated with increased PFS (RR for fixed effect $=0.9$ [0.83-0.98]) (Figure 2). There was no significant publication bias $(p=0.79)$ (Supplementary Figure S1).

After a median follow-up ranging from 16 to 108 months, 1453 deaths had occurred. Regarding overall survival, because of significant heterogeneity in the overall analysis $\left(\mathrm{I}^{2}=45 \%, p=0.01\right)$ and no significant fixed or random effect (Supplementary Figure S2), we further pooled studies according to dose-intense cisplatin-based neoadjuvant chemotherapy. Dose-intense chemotherapy was calculated as the ratio of the total dose of cisplatin $\left(\mathrm{mg} / \mathrm{m}^{2}\right) /$ chemotherapy duration (weeks) and was expressed in $\mathrm{mg} / \mathrm{m}^{2} / 3$ weeks. Doseintense cisplatin administration ranged from 50 to $160 \mathrm{mg} / \mathrm{m}^{2} / 3$ weeks with a median value of $72.5 \mathrm{mg} / \mathrm{m}^{2} / 3$ weeks. After a sensitivity analysis using the threshold of 72.5 , and then $105 \mathrm{mg} / \mathrm{m}^{2} / 3$ weeks, we classified studies in a binary manner as a function of dose-intense cisplatin use (yes $/$ no) at the chosen thresholds (i.e., $<$ or $\geq$ ) to maximize both fixed and random effects and to reduce heterogeneity. Dose-intense cisplatin $\geq 72.5 \mathrm{mg} / \mathrm{m}^{2} / 3$ weeks was significantly associated with OS (RR for fixed effect $=0.87$ [0.76-0.98]). In contrast, a dose-intense cisplatin $<72.5 \mathrm{mg} / \mathrm{m}^{2} / 3$ weeks was significantly deleterious ( $R R=1.15$ [1.02-1.28]) (Supplementary Figure S3). The benefit was even greater at dose-intense cisplatin $\geq 105 \mathrm{mg} / \mathrm{m}^{2} / 3$ weeks $(\mathrm{RR}$ for fixed effect $=0.79$ [0.67-0.93]) (Figure 3). 


\section{Study}

da Costa SCS. et al 2019

Yang Z et al. 2016

Katsumata N et al. 2013

Mossa B et al. 2010

Chen $\mathrm{H}$ et al. 2008

Eddy GL et al. 2007

Cai HB et al. 2006

Tabata T et al. 2003

Benedetti-Panici P et al. 2002

Chang TC et al. 2000

Symonds RP et al. 2000

Sardi JE et al. 1997

Sundfor K et al. 1996

Kigawa J et al. 1996

Tattersall MH et al. 1995

Chauvergne J et al. 1993

Tattersall MH et al. 1992

Souhami L et al. 1991

\section{Experimental Control \\ Events Total Events Tota}

$\begin{array}{rrrr}30 & 55 & 20 & 52 \\ 10 & 109 & 6 & 110 \\ 27 & 67 & 25 & 67 \\ 55 & 159 & 60 & 129 \\ 17 & 72 & 26 & 70 \\ 63 & 145 & 62 & 143 \\ 9 & 52 & 14 & 54 \\ 18 & 32 & 15 & 29 \\ 84 & 227 & 100 & 214 \\ 21 & 68 & 14 & 52 \\ 62 & 100 & 78 & 104 \\ 8 & 102 & 18 & 103 \\ 30 & 47 & 33 & 47 \\ 8 & 25 & 15 & 25 \\ 53 & 129 & 46 & 131 \\ 45 & 75 & 49 & 76 \\ 18 & 34 & 20 & 37 \\ 22 & 39 & 30 & 52 \\ & & & \\ & 1537 & & 1495\end{array}$

495
Risk Ratio

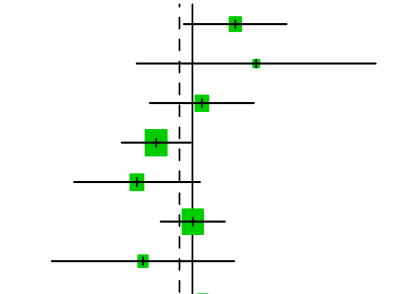

\section{RR $\quad 95 \%-\mathrm{Cl}$ Weight}

$1.42[0.93 ; 2.16] \quad 3.2 \%$

$1.68[0.63 ; 4.47] \quad 0.9 \%$

$1.08[0.71 ; 1.65] \quad 3.9 \%$

$0.74[0.56 ; 0.99] \quad 10.4 \%$

$0.64[0.38 ; 1.06] \quad 4.1 \%$

$1.00[0.77 ; 1.30] \quad 9.8 \%$

$0.67[0.32 ; 1.41] \quad 2.2 \%$

$1.09[0.68 ; 1.73] \quad 2.5 \%$

$0.79[0.63 ; 0.99] \quad 16.2 \%$

$1.15[0.65 ; 2.03] \quad 2.5 \%$

$0.83[0.68 ; 1.00] \quad 12.0 \%$

$0.45[0.20 ; 0.99] \quad 2.8 \%$

$0.91[0.68 ; 1.21] \quad 5.2 \%$

$0.53[0.28 ; 1.03] \quad 2.4 \%$

$1.17[0.86 ; 1.60] \quad 7.2 \%$

$0.93[0.73 ; 1.19] \quad 7.6 \%$

$0.98[0.63 ; 1.51] \quad 3.0 \%$

$0.98[0.68 ; 1.40] \quad 4.0 \%$

$0.90[0.83 ; 0.98] 100.0 \%$

Fixed effect model
Heterogeneity: $I^{2}=28 \%, \tau^{2}=0.0120, p=0.13$

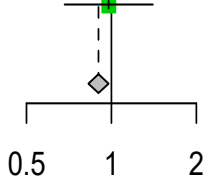

Figure 2. Forest plot of randomized clinical trials assessing predictive value of cisplatin-based neoadjuvant chemotherapy on progression-free survival in locally advanced cervical cancers. Relative risks (RR) are given with $95 \%$ confidence intervals. Overall $R R=0.9$ (95\% CI, 0.83 to 0.98$)$; heterogeneity $\mathrm{I}^{2}=0.28, p=0.13$.

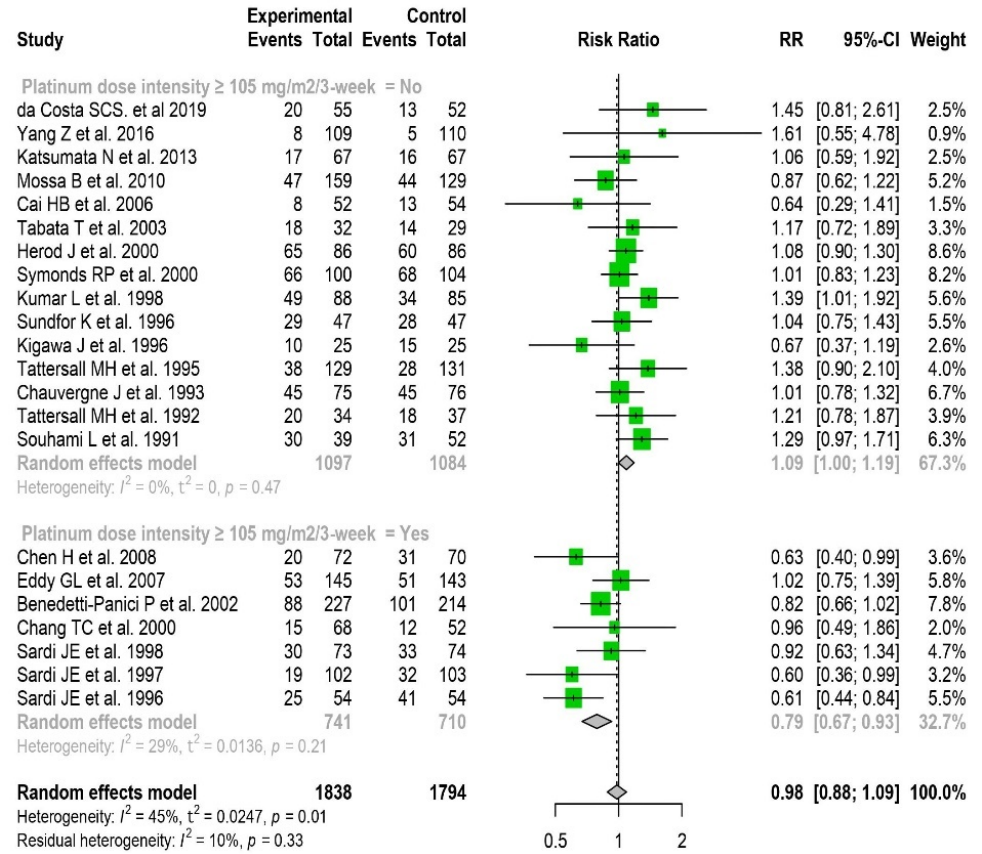

Figure 3. Forest plot of randomized clinical trials assessing the predictive value of neoadjuvant cisplatin-based chemotherapy for overall survival in locally advanced cervical cancer. Relative risks (RR) are given with $95 \%$ confidence intervals. Neoadjuvant chemotherapy using dose-intense cisplatin-based chemotherapy $\geq 105 \mathrm{mg} / \mathrm{m}^{2} / 3$ weeks significantly improves overall survival: $\mathrm{RR}=0.79(95 \% \mathrm{CI}, 0.67$ to 0.93$)$; heterogeneity $\mathrm{I}^{2}=29 \%, p=0.21$. 
When we restricted the analysis to subgroups of patients whose local treatment was similar (surgery or radiotherapy), dose-intense cisplatin was beneficial in both cases and more marked when local treatment was limited to a surgery (Supplementary Figures S4 and S5).

In contrast, when we pooled studies according to (i) triplet vs. not triplet cisplatin-based neoadjuvant chemotherapy or (ii) duration of chemotherapy ( $\leq$ vs. $>6$ weeks), there was no significant difference in terms of overall survival (Supplementary Figures S6 and S7).

For PFS, we performed the same subgroup analyses. In particular, the benefit of using cisplatin-based neoadjuvant chemotherapy was also significantly increased when cisplatin dose-intensity was higher than $72.5 \mathrm{mg} / \mathrm{m}^{2} / 3$ weeks ( $R R$ for fixed effect $=0.86$ [0.78-0.95]). The RR of 0.84 was comparable in terms of PFS for the subgroup of studies with surgery as local treatment and receiving neoadjuvant chemotherapy, despite that it did not reach statistical significance. There was no heterogeneity between the subgroups (Supplementary Figures S8 and S9).

Table 2 shows the RR for fixed effect in the sensitivity analysis. There was no significant publication bias ( $p=0.73$ ) (Supplementary Figure S10), and the overall quality of the studies included in this meta-analysis was good according to the Cochrane Collaboration Risk of Bias tool (Supplementary Figures S11 and S12). In all, more than 50\% of the randomized clinical trials were at low risk of bias.

Table 2. Sensitivity analysis of randomized clinical trials assessing the predictive value of cisplatinbased neoadjuvant chemotherapy for overall survival.

\begin{tabular}{ccc}
\hline & RR for Fixed Effect & [95\%CI] \\
\hline Overall analysis & 0.97 & {$[0.90-1.05]$} \\
Dose-intense cisplatin $\geq 72.5 \mathrm{mg} / \mathrm{m}^{2} / 3$ weeks & 0.87 & {$[0.76-0.98]$} \\
Dose-intense cisplatin $\geq 105 \mathrm{mg} / \mathrm{m}^{2} / 3$ weeks & 0.79 & {$[0.67-0.93]$} \\
Triplet cisplatin-based chemotherapy (yes) & 0.97 & {$[0.83-1.13]$} \\
Chemotherapy duration $(\leq 6$ weeks) & 0.91 & {$[0.80 ; 1.04]$} \\
\hline
\end{tabular}

RR, relative risk; CI, confidence interval.

Overall, dose-intense cisplatin-based neoadjuvant chemotherapy was significantly associated with a survival benefit in the treatment of locally advanced cervical carcinoma, and the higher the dose, the greater the survival benefit.

\section{Discussion}

For the first time, our meta-analysis has demonstrated the benefit of using dose-intense cisplatin-based neoadjuvant chemotherapy for the treatment of locally advanced cervical cancer. Two meta-analyses had previously been conducted in this setting. In 2020, the second meta-analysis only focused on stages IB2 and II cervical cancers and was thus limited to 5 studies, including 2 case-control studies and only 1275 patients, with a nonsignificant trend for a $40 \%$ reduction in the risk of death with neoadjuvant chemotherapy [50]. In 2003, the first meta-analysis reported data for 2074 women without evidencing any benefit of neoadjuvant chemotherapy. However, when the authors analyzed studies using doseintense cisplatin separately for doses over $25 \mathrm{mg} / \mathrm{m}^{2} /$ week (i.e., over $75 \mathrm{mg} / \mathrm{m}^{2} / 3$ weeks), there was a nonsignificant trend towards a survival benefit $(\mathrm{HR}=0.91 ; p=0.2)[14]$. In our meta-analysis, using dose-intense cisplatin at a dose over $72.5 \mathrm{mg} / \mathrm{m}^{2} / 3$ weeks, the RR for overall survival was $0.87(p<0.05)$. Using dose-intense cisplatin at doses over $105 \mathrm{mg} / \mathrm{m}^{2} / 3$ weeks, the benefit was even greater with a RR of 0.79 . In contrast, using cisplatin at a dose less than $72.5 \mathrm{mg} / \mathrm{m}^{2} / 3$ weeks was significantly associated with shorter survival, possibly related to the delay in instating local treatment. This was also true for PFS. Since 2003, 8 additional published trials have been reported, corresponding to 1345 additional patients, enabling the statistical power of our meta-analysis to be increased.

The stringent methodology is a strength of our meta-analysis on published data, with clearly defined PICOS criteria, careful selection of the clinical trials finally retained, absence of heterogeneity across the subgroups compared, and quality control of the 22 studies to ensure the absence of publication bias. A limitation of this meta-analysis could be linked to 
the local treatments instated after neoadjuvant chemotherapy, since radiotherapy combined with concomitant chemotherapy, the standard care, only concerned one clinical trial. Most local treatments were either surgery alone or radiotherapy alone. However, dose-intense cisplatin-based neoadjuvant chemotherapy remained beneficial when we analyzed these subgroups separately, and there was no heterogeneity between the subgroups of patients receiving or not receiving cisplatin-based dose-intense neoadjuvant chemotherapy, independently from the local treatment performed after neoadjuvant chemotherapy. In particular, two clinical trials included in this meta-analysis raise the concern that local treatment instated after neoadjuvant chemotherapy was not the same between the experimental arm and the control arm: the studies by Benedetti-Panici et al. [40] and Chang et al. [41]. Even after exclusion of these two trials from our meta-analysis, the results were not different with a survival benefit of using dose-intense cisplatin-based neoadjuvant chemotherapy (Supplementary Figure S13).

In addition, one should keep in mind that radiotherapy is not available in many countries. This is particularly true in low-income countries, accounting for more than $80 \%$ of cervical cancers diagnosed worldwide, where the incidence of locally advanced cervical cancer is the highest. In these countries, surgery is a frequent therapeutic alternative to chemoradiotherapy when it is not available [5,6,51], and the American Association Society has recommended that "In basic settings where patients cannot be treated with radiation therapy, extrafascial hysterectomy either alone or after chemotherapy may be an option for women with stage IA1 to IVA cervical cancer" [10]. This is also true in upper- and middle-income countries when daily access to radiotherapy is difficult due to long travel distances [8,9]. In all these cases, cisplatin-based dose-intense neoadjuvant chemotherapy could be beneficial when local treatment is suboptimal, both in terms of PFS and OS. In a previous meta-analysis, Ye et al. demonstrated the benefit of neoadjuvant chemotherapy when local treatment was surgery [50]. One should keep in mind that when surgery is chosen as a local treatment for cervical cancer with high-risk characteristics, adjuvant radiotherapy should be proposed as standard care despite that its access may be often very difficult [10].

One concern may be the clinical and limited toxicities when using high doses of cisplatin. These toxicities, mainly hematological and gastrointestinal toxicities, are usually easily manageable using new-generation antiemetics and granulocyte colony-stimulating factor (G-CSF) [52-54].

In metastatic stages, triplet regimens of chemotherapy lead to higher response rates compared to doublet regimens and to monotherapy, but survival data were insufficient to reach any relevant conclusion [16]. In our meta-analysis, we did not find any survival benefit of using a cisplatin-based triplet regimen for neoadjuvant chemotherapy in locally advanced cervical cancer. Further studies are required to assess this point, including the addition of a taxane to cisplatin, since only one clinical trial has evaluated the combination of paclitaxel and carboplatin in the neoadjuvant setting, evidencing no clear benefit [55].

\section{Conclusions}

Even though radiotherapy combined with weekly cisplatin-based chemotherapy remains standard of care for the treatment of locally advanced cervical cancer, our metaanalysis makes it possible to consider the use of dose-intense cisplatin-based neoadjuvant chemotherapy when local treatment is suboptimal and opens perspectives for designing new clinical trials in this setting, particularly for FIGO stages III and IVA. Neoadjuvant chemotherapy could be proposed when surgery is local treatment instead of standard chemoradiation therapy for the treatment of locally advanced cervical cancer, as proposed in the EORTC 55994 trial design [56] (Figure 4). 


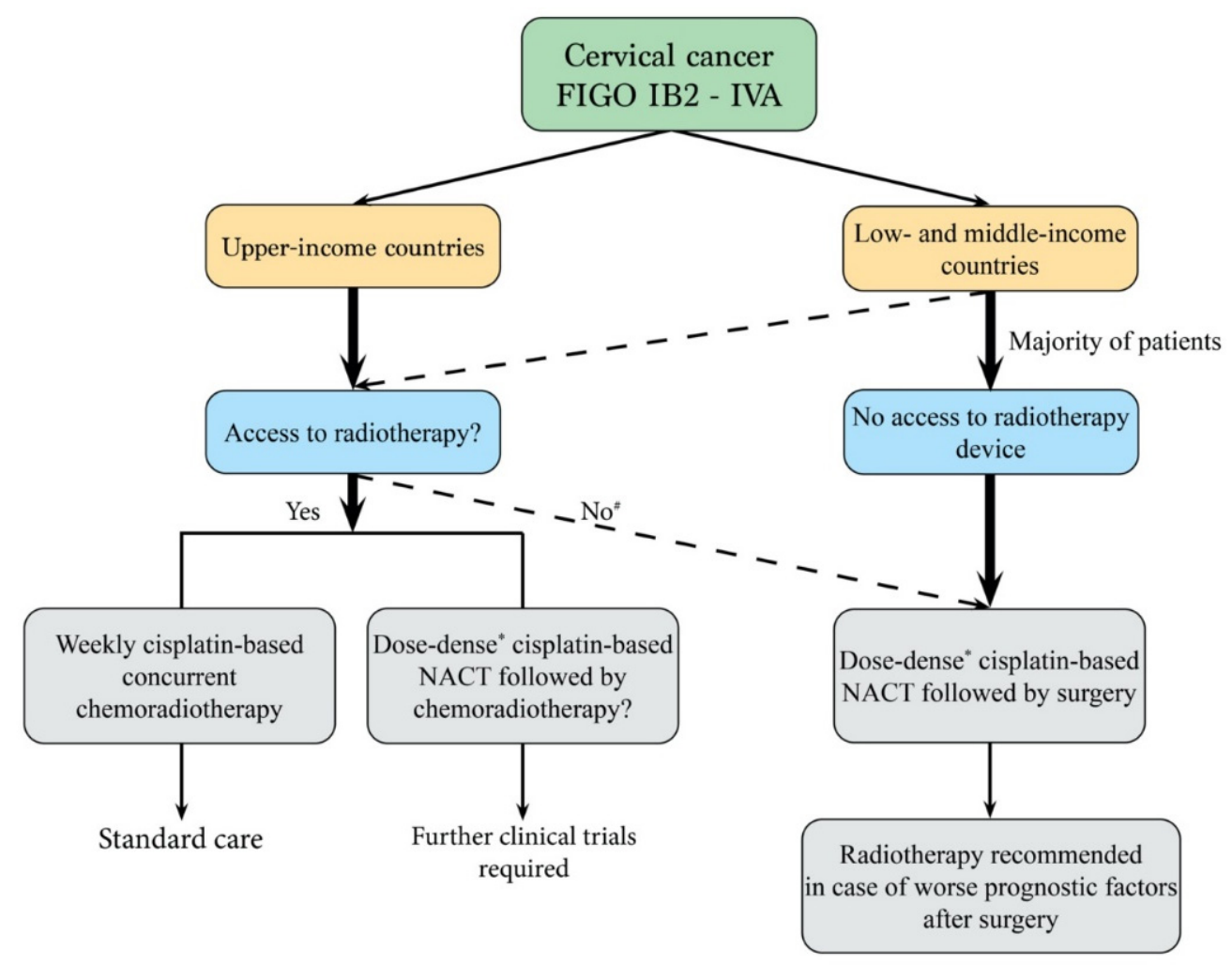

Figure 4. Decision tree for the treatment of locally advanced cervical cancer. NACT, neoadjuvant chemotherapy; \# vulnerable populations, long travel distances; * minimal cisplatin dose of $75 \mathrm{mg} / \mathrm{m}^{2} / 3$ weeks.

Supplementary Materials: The following supporting information can be downloaded at: https: / / www.mdpi.com/article/10.3390/cancers14030842/s1. Figure S1: Funnel plot of risk of publication bias in randomized clinical trials published between 1991 and 2019; Figure S2: Forest plot for OS; Figure S3: Forest plot for OS as function of cisplatin $\geq 72.5 \mathrm{mg} / \mathrm{m}^{2} / 3$ weeks; Figure S4: Forest plot for OS as function of cisplatin $\geq 75 \mathrm{mg} / \mathrm{m}^{2} / 3$ weeks with local treatment subgroup as surgery; Figure S5: Forest plot for OS as function of cisplatin $\geq 105 \mathrm{mg} / \mathrm{m}^{2} / 3$ weeks with local treatment subgroup as radiotherapy; Figure S6: Forest plot for OS as function of triplet chemotherapy. The subgroup analysis does not evidence any significant benefit of using a triplet regimen during neoadjuvant; Figure S7: Forest plot for OS as function of chemotherapy duration; Figure S8: Forest plot for PFS as function of cisplatin dose-intensity $\geq 72.5 \mathrm{mg} / \mathrm{m}^{2} / 3$ weeks; Figure S9: Forest plot for PFS as function of surgery as local treatment; Figure S10: Funnel plot of risk of publication bias in randomized clinical trials published between 1991 and 2019 assessing OS in locally advanced cervical cancer; Figure S11: Risk of bias assessments for the 22 studies included in a Cochrane review; Figure S12: Overall assessment of risk of bias: graph for the 22 studies included in this meta-analysis; Figure S13: Forest plot for OS as function of cisplatin $\geq 105 \mathrm{mg} / \mathrm{m}^{2} / 3$ weeks after exclusion two studies (Benedetti-Panici P 2002 and Chang TC 2000); Table S1: Eligibility using the PICOS criteria; Table S2: Articles excluded at full-text screening stage.

Author Contributions: Conception and design: G.B., F.P. and V.T.N. Administrative support: G.B. Collection and assembly of data: S.W., L.Z., A.B., M.P., V.T.N., F.P. and G.B. Data analysis and interpretation: M.P., V.T.N., F.P. and G.B. Manuscript draft: V.T.N. and G.B. All authors have read and agreed to the published version of the manuscript.

Funding: This work was supported by AP-HP International Relationships, INSERM.

Institutional Review Board Statement: Not applicable.

Informed Consent Statement: Not applicable.

Data Availability Statement: All the data presented in this study are identified within the text, tables, and figures. 
Acknowledgments: We thank Angela Swaine for the revision of English language.

Conflicts of Interest: These authors have no conflict of interest to report.

\section{Abbreviation}

$\begin{array}{ll}\text { ERCC1 } & \text { Excision Repair Cross-Complementation group } 1 \\ \text { PARP1 } & \text { Poly (ADP-ribose) Polymerase 1 } \\ \text { HSPB1/p } & \text { Heat Shock Protein Family B member 1 phosphorylated } \\ \text { PRISMA } & \text { Preferred Reporting Items for Systematic reviews and Meta-Analyses } \\ \text { PROSPERO } & \text { International Prospective Register of Systematic Review } \\ \text { MeSH } & \text { Medical Subject Headings } \\ \text { PICOS } & \text { Population, Intervention, Comparator group, Outcomes, and Study design } \\ \text { CHSRI } & \text { Cochrane Handbook for Systematic Reviews of Interventions } \\ \text { NACT } & \text { Neoadjuvant Chemotherapy }\end{array}$

\section{References}

1. Sung, H.; Ferlay, J.; Siegel, R.L.; Laversanne, M.; Soerjomataram, I.; Jemal, A.; Bray, F. Global Cancer Statistics 2020: GLOBOCAN Estimates of Incidence and Mortality Worldwide for 36 Cancers in 185 Countries. CA Cancer J. Clin. 2021, 71, 209-249. [CrossRef] [PubMed]

2. Quinn, M.; Benedet, J.; Odicino, F.; Maisonneuve, P.; Beller, U.; Creasman, W.; Heintz, A.P.M.; Ngan, H.Y.S.; Pecorelli, S. Carcinoma of the Cervix Uteri. FIGO 26th Annual Report on the Results of Treatment in Gynecological Cancer. Int. J. Gynaecol. Obstet. Off. Organ Int. Fed. Gynaecol. Obstet. 2006, 95 (Suppl. S1), 43-103. [CrossRef]

3. Gennigens, C.; De Cuypere, M.; Hermesse, J.; Kridelka, F.; Jerusalem, G. Optimal Treatment in Locally Advanced Cervical Cancer. Expert Rev. Anticancer Ther. 2021, 21, 657-671. [CrossRef] [PubMed]

4. Datta, N.R.; Stutz, E.; Liu, M.; Rogers, S.; Klingbiel, D.; Siebenhüner, A.; Singh, S.; Bodis, S. Concurrent Chemoradiotherapy vs. Radiotherapy Alone in Locally Advanced Cervix Cancer: A Systematic Review and Meta-Analysis. Gynecol. Oncol. 2017, 145, 374-385. [CrossRef]

5. LaVigne, A.W.; Triedman, S.A.; Randall, T.C.; Trimble, E.L.; Viswanathan, A.N. Cervical Cancer in Low and Middle Income Countries: Addressing Barriers to Radiotherapy Delivery. Gynecol. Oncol. Rep. 2017, 22, 16-20. [CrossRef]

6. Grover, S.; Xu, M.J.; Yeager, A.; Rosman, L.; Groen, R.S.; Chackungal, S.; Rodin, D.; Mangaali, M.; Nurkic, S.; Fernandes, A.; et al. A Systematic Review of Radiotherapy Capacity in Low- and Middle-Income Countries. Front. Oncol. 2015, 4, 380. [CrossRef]

7. Randall, T.C.; Ghebre, R. Challenges in Prevention and Care Delivery for Women with Cervical Cancer in Sub-Saharan Africa. Front. Oncol. 2016, 6, 160. [CrossRef]

8. Thompson, S.C.; Cheetham, S.; Baxi, S. The Enablers, Barriers and Preferences of Accessing Radiation Therapy Facilities in the Rural Developed World-A Systematic Review. BMC Cancer 2017, 17, 794. [CrossRef]

9. Mendez, L.C.; Moraes, F.Y.; Castilho, M.S.; Louie, A.V.; Qu, X.M. Lives and Economic Loss in Brazil Due to Lack of Radiotherapy Access in Cervical Cancer: A Cost-Effectiveness Analysis. Clin. Oncol. 2019, 31, e143-e148. [CrossRef]

10. Chuang, L.T.; Temin, S.; Camacho, R.; Dueñas-Gonzalez, A.; Feldman, S.; Gultekin, M.; Gupta, V.; Horton, S.; Jacob, G.; Kidd, E.A.; et al. Management and Care of Women With Invasive Cervical Cancer: American Society of Clinical Oncology Resource-Stratified Clinical Practice Guideline. J. Glob. Oncol. 2016, 2, 311-340. [CrossRef]

11. Mieog, J.S.D.; van der Hage, J.A.; van de Velde, C.J.H. Neoadjuvant Chemotherapy for Operable Breast Cancer. Br. J. Surg. 2007, 94, 1189-1200. [CrossRef] [PubMed]

12. Korde, L.A.; Somerfield, M.R.; Carey, L.A.; Crews, J.R.; Denduluri, N.; Hwang, E.S.; Khan, S.A.; Loibl, S.; Morris, E.A.; Perez, A.; et al. Neoadjuvant Chemotherapy, Endocrine Therapy, and Targeted Therapy for Breast Cancer: ASCO Guideline. J. Clin. Oncol. 2021, 39, 1485-1505. [CrossRef] [PubMed]

13. Du Bois, A.; Baert, T.; Vergote, I. Role of Neoadjuvant Chemotherapy in Advanced Epithelial Ovarian Cancer. J. Clin. Oncol. 2019, 37, 2398-2405. [CrossRef] [PubMed]

14. NACCCMA Collaboration Neoadjuvant Chemotherapy for Locally Advanced Cervical Cancer: A Systematic Review and Meta-Analysis of Individual Patient Data from 21 Randomised Trials. Eur. J. Cancer 2003, 39, 2470-2486. [CrossRef]

15. Reducing Uncertainties About the Effects of Chemoradiotherapy for Cervical Cancer: A Systematic Review and Meta-Analysis of Individual Patient Data From 18 Randomized Trials. J. Clin. Oncol. 2008, 26, 5802-5812. [CrossRef]

16. Scatchard, K.; Forrest, J.; Flubacher, M.; Cornes, P.; Williams, C. Chemotherapy for Metastatic and Recurrent Cervical Cancer. Cochrane Database Syst. Rev. 2012, 10, CD006469. [CrossRef]

17. Basu, A.; Krishnamurthy, S. Cellular Responses to Cisplatin-Induced DNA Damage. J. Nucleic Acids 2010, 2010, 201367. [CrossRef]

18. Chen, S.-H.; Huang, W.-T.; Kao, W.-C.; Hsiao, S.-Y.; Pan, H.-Y.; Fang, C.-W.; Shiue, Y.-L.; Chou, C.-L.; Li, C.-F. O6-MethylguanineDNA Methyltransferase Modulates Cisplatin-Induced DNA Double-Strand Breaks by Targeting the Homologous Recombination Pathway in Nasopharyngeal Carcinoma. J. Biomed. Sci. 2021, 28, 2. [CrossRef] 
19. Konstantinopoulos, P.A.; Ceccaldi, R.; Shapiro, G.I.; D'Andrea, A.D. Homologous Recombination Deficiency: Exploiting the Fundamental Vulnerability of Ovarian Cancer. Cancer Discov. 2015, 5, 1137-1154. [CrossRef]

20. Telli, M.L.; Timms, K.M.; Reid, J.; Hennessy, B.; Mills, G.B.; Jensen, K.C.; Szallasi, Z.; Barry, W.T.; Winer, E.P.; Tung, N.M.; et al Homologous Recombination Deficiency (HRD) Score Predicts Response to Platinum-Containing Neoadjuvant Chemotherapy in Patients with Triple-Negative Breast Cancer. Clin. Cancer Res. 2016, 22, 3764. [CrossRef]

21. Chen, Q.; Cai, D.; Li, M.; Wu, X. The Homologous Recombination Protein RAD51 Is a Promising Therapeutic Target for Cervical Carcinoma. Oncol. Rep. 2017, 38, 767-774. [CrossRef] [PubMed]

22. Zhu, J.; Ji, S.; Hu, Q.; Chen, Q.; Liu, Z.; Wu, J.; Gu, K. The Prognostic Value of Excission Repair Cross-Complementation Group One Enzyme Expression in Locally Advanced Cervical Carcinoma Patients Treated with Cisplatin-Based Treatment: A Meta-Analysis. Int. J. Gynecol. Cancer 2019, 29, 35. [CrossRef] [PubMed]

23. Prasad, C.B.; Prasad, S.B.; Yadav, S.S.; Pandey, L.K.; Singh, S.; Pradhan, S.; Narayan, G. Olaparib Modulates DNA Repair Efficiency, Sensitizes Cervical Cancer Cells to Cisplatin and Exhibits Anti-Metastatic Property. Sci. Rep. 2017, 7, 12876. [CrossRef] [PubMed]

24. Real, N.E.; Castro, G.N.; Darío Cuello-Carrión, F.; Perinetti, C.; Röhrich, H.; Cayado-Gutiérrez, N.; Guerrero-Gimenez, M.E.; Ciocca, D.R. Molecular Markers of DNA Damage and Repair in Cervical Cancer Patients Treated with Cisplatin Neoadjuvant Chemotherapy: An Exploratory Study. Cell Stress Chaperones 2017, 22, 811-822. [CrossRef]

25. Liberati, A.; Altman, D.G.; Tetzlaff, J.; Mulrow, C.; Gøtzsche, P.C.; Ioannidis, J.P.A.; Clarke, M.; Devereaux, P.J.; Kleijnen, J.; Moher, D. The PRISMA Statement for Reporting Systematic Reviews and Meta-Analyses of Studies That Evaluate Health Care Interventions: Explanation and Elaboration. PLoS Med. 2009, 6, e1000100. [CrossRef]

26. Higgins, J.; Thomas, J.; Chandler, J. Cochrane Handbook for Systematic Reviews of Interventions, 2nd ed.; John Wiley \& Sons: Chichester, UK, 2019; pp. 194-241.

27. Sardi, J.; Giaroli, A.; Sananes, C.; Rueda, N.G.; Vighi, S.; Ferreira, M.; Bastardas, M.; Paniceres, G.; Di Paola, G. Randomized Trial with Neoadjuvant Chemotherapy in Stage IIIB Squamous Carcinoma Cervix Uteri: An Unexpected Therapeutic Management. Int. J. Gynecol. Cancer 1996, 6, 85-93. [CrossRef]

28. Sardi, J.E.; Giaroli, A.; Sananes, C.; Ferreira, M.; Soderini, A.; Bermudez, A.; Snaidas, L.; Vighi, S.; Gomez Rueda, N.; di Paola, G. Long-Term Follow-up of the First Randomized Trial Using Neoadjuvant Chemotherapy in Stage Ib Squamous Carcinoma of the Cervix: The Final Results. Gynecol. Oncol. 1997, 67, 61-69. [CrossRef] [PubMed]

29. Sardi; Sananes; Giaroli; Bermúdez; Ferreira; Soderini; Snaidas; Guardado; Anchezar; Ortiz, C.; et al. Neoadjuvant Chemotherapy in Cervical Carcinoma Stage IIB: A Randomized Controlled Trial. Int. J. Gynecol. Cancer 1998, 8, 441-450. [CrossRef]

30. Tattersall, M.H.; Lorvidhaya, V.; Vootiprux, V.; Cheirsilpa, A.; Wong, F.; Azhar, T.; Lee, H.P.; Kang, S.B.; Manalo, A.; Yen, M.S. Randomized Trial of Epirubicin and Cisplatin Chemotherapy Followed by Pelvic Radiation in Locally Advanced Cervical Cancer. Cervical Cancer Study Group of the Asian Oceanian Clinical Oncology Association. J. Clin. Oncol. Off. J. Am. Soc. Clin. Oncol. 1995, 13, 444-451. [CrossRef]

31. Tattersall, M.H.N.; Ramirez, C.; Coppleson, M. A Randomized Trial Comparing Platinum-Based Chemotherapy Followed by Radiotherapy vs. Radiotherapy Alone in Patients with Locally Advanced Cervical Cancer. Int. J. Gynecol. Cancer Off. J. Int. Gynecol. Cancer Soc. 1992, 2, 244-251. [CrossRef] [PubMed]

32. Da Costa, S.C.S.; Bonadio, R.C.; Gabrielli, F.C.G.; Aranha, A.S.; Dias Genta, M.L.N.; Miranda, V.C.; de Freitas, D.; Abdo Filho, E.; Ferreira, P.A.O.; Machado, K.K.; et al. Neoadjuvant Chemotherapy With Cisplatin and Gemcitabine Followed by Chemoradiation Versus Chemoradiation for Locally Advanced Cervical Cancer: A Randomized Phase II Trial. J. Clin. Oncol. 2019, 37, 3124-3131. [CrossRef]

33. Yang, Z.; Chen, D.; Zhang, J.; Yao, D.; Gao, K.; Wang, H.; Liu, C.; Yu, J.; Li, L. The Efficacy and Safety of Neoadjuvant Chemotherapy in the Treatment of Locally Advanced Cervical Cancer: A Randomized Multicenter Study. Gynecol. Oncol. 2016, 141, 231-239. [CrossRef]

34. Katsumata, N.; Yoshikawa, H.; Kobayashi, H.; Saito, T.; Kuzuya, K.; Nakanishi, T.; Yasugi, T.; Yaegashi, N.; Yokota, H.; Kodama, S.; et al. Phase III Randomised Controlled Trial of Neoadjuvant Chemotherapy plus Radical Surgery vs Radical Surgery Alone for Stages IB2, IIA2, and IIB Cervical Cancer: A Japan Clinical Oncology Group Trial (JCOG 0102). Br. J. Cancer 2013, 108, 1957-1963. [CrossRef]

35. Mossa, B.; Mossa, S.; Corosu, L.; Marziani, R. Follow-up in a Long-Term Randomized Trial with Neoadjuvant Chemotherapy for Squamous Cell Cervical Carcinoma. Eur. J. Gynaecol. Oncol. 2010, 31, 497-503.

36. Chen, H.; Liang, C.; Zhang, L.; Huang, S.; Wu, X. Clinical Efficacy of Modified Preoperative Neoadjuvant Chemotherapy in the Treatment of Locally Advanced (Stage IB2 to IIB) Cervical Cancer: Randomized Study. Gynecol. Oncol. 2008, 110, 308-315. [CrossRef]

37. Eddy, G.L.; Bundy, B.N.; Creasman, W.T.; Spirtos, N.M.; Mannel, R.S.; Hannigan, E.; O'Connor, D. Treatment of (“bulky”) Stage IB Cervical Cancer with or without Neoadjuvant Vincristine and Cisplatin Prior to Radical Hysterectomy and Pelvic/Para-Aortic Lymphadenectomy: A Phase III Trial of the Gynecologic Oncology Group. Gynecol. Oncol. 2007, 106, 362-369. [CrossRef]

38. Cai, H.-B.; Chen, H.-Z.; Yin, H.-H. Randomized Study of Preoperative Chemotherapy versus Primary Surgery for Stage IB Cervical Cancer. J. Obstet. Gynaecol. Res. 2006, 32, 315-323. [CrossRef]

39. Tabata, T.; Takeshima, N.; Nishida, H.; Hirai, Y.; Hasumi, K. A Randomized Study of Primary Bleomycin, Vincristine, Mitomycin and Cisplatin (BOMP) Chemotherapy Followed by Radiotherapy versus Radiotherapy Alone in Stage IIIB and IVA Squamous Cell Carcinoma of the Cervix. Anticancer Res. 2003, 23, 2885-2890. 
40. Benedetti-Panici, P.; Greggi, S.; Colombo, A.; Amoroso, M.; Smaniotto, D.; Giannarelli, D.; Amunni, G.; Raspagliesi, F.; Zola, P.; Mangioni, C.; et al. Neoadjuvant Chemotherapy and Radical Surgery versus Exclusive Radiotherapy in Locally Advanced Squamous Cell Cervical Cancer: Results from the Italian Multicenter Randomized Study. J. Clin. Oncol. Off. J. Am. Soc. Clin. Oncol. 2002, 20, 179-188. [CrossRef]

41. Chang, T.C.; Lai, C.H.; Hong, J.H.; Hsueh, S.; Huang, K.G.; Chou, H.H.; Tseng, C.J.; Tsai, C.S.; Chang, J.T.; Lin, C.T.; et al. Randomized Trial of Neoadjuvant Cisplatin, Vincristine, Bleomycin, and Radical Hysterectomy versus Radiation Therapy for Bulky Stage IB and IIA Cervical Cancer. J. Clin. Oncol. Off. J. Am. Soc. Clin. Oncol. 2000, 18, 1740-1747. [CrossRef] [PubMed]

42. Herod, J.; Burton, A.; Buxton, J.; Tobias, J.; Luesley, D.; Jordan, S.; Dunn, J.; Poole, C.J. A Randomised, Prospective, Phase III Clinical Trial of Primary Bleomycin, Ifosfamide and Cisplatin (BIP) Chemotherapy Followed by Radiotherapy versus Radiotherapy Alone in Inoperable Cancer of the Cervix. Ann. Oncol. Off. J. Eur. Soc. Med. Oncol. 2000, 11, 1175-1181. [CrossRef] [PubMed]

43. Symonds, R.P.; Habeshaw, T.; Reed, N.S.; Paul, J.; Pyper, E.; Yosef, H.; Davis, J.; Hunter, R.; Davidson, S.E.; Stewart, A.; et al. The Scottish and Manchester Randomised Trial of Neo-Adjuvant Chemotherapy for Advanced Cervical Cancer. Eur. J. Cancer Oxf. Engl. 1990 2000, 36, 994-1001. [CrossRef]

44. Kumar, L.; Grover, R.; Pokharel, Y.H.; Chander, S.; Kumar, S.; Singh, R.; Rath, G.K.; Kochupillai, V. Neoadjuvant Chemotherapy in Locally Advanced Cervical Cancer: Two Randomised Studies. Aust. N. Z. J. Med. 1998, 28, 387-390. [CrossRef] [PubMed]

45. Sundfør, K.; Tropé, C.G.; Högberg, T.; Onsrud, M.; Koern, J.; Simonsen, E.; Bertelsen, K.; Westberg, R. Radiotherapy and Neoadjuvant Chemotherapy for Cervical Carcinoma. A Randomized Multicenter Study of Sequential Cisplatin and 5-Fluorouracil and Radiotherapy in Advanced Cervical Carcinoma Stage 3B and 4A. Cancer 1996, 77, 2371-2378. [CrossRef]

46. Kigawa, J.; Minagawa, Y.; Ishihara, H.; Itamochi, H.; Kanamori, Y.; Terakawa, N. The Role of Neoadjuvant Intraarterial Infusion Chemotherapy with Cisplatin and Bleomycin for Locally Advanced Cervical Cancer. Am. J. Clin. Oncol. 1996, 19, 225-259. [CrossRef]

47. Chauvergne, J.; Lhommé, C.; Rohart, J.; Héron, J.F.; Ayme, Y.; Goupil, A.; Fargeot, P.; David, M. [Neoadjuvant chemotherapy of stage IIb or III cancers of the uterine cervix. Long-term results of a multicenter randomized trial of 151 patients]. Bull. Cancer 1993, 80, 1069-1079. [PubMed]

48. Souhami, L.; Gil, R.A.; Allan, S.E.; Canary, P.C.; Araújo, C.M.; Pinto, L.H.; Silveira, T.R. A Randomized Trial of Chemotherapy Followed by Pelvic Radiation Therapy in Stage IIIB Carcinoma of the Cervix. J. Clin. Oncol. Off. J. Am. Soc. Clin. Oncol. 1991, 9, 970-977. [CrossRef]

49. Bhatla, N.; Berek, J.S.; Cuello Fredes, M.; Denny, L.A.; Grenman, S.; Karunaratne, K.; Kehoe, S.T.; Konishi, I.; Olawaiye, A.B.; Prat, J.; et al. Revised FIGO Staging for Carcinoma of the Cervix Uteri. Int. J. Gynaecol. Obstet. Off. Organ Int. Fed. Gynaecol. Obstet. 2019, 145, 129-135. [CrossRef]

50. Ye, Q.; Yang, Y.; Tang, X.; Li, J.; Li, X.; Zhang, Y. Neoadjuvant Chemotherapy Followed by Radical Surgery versus Radiotherapy (with or without Chemotherapy) in Patients with Stage IB2, IIA, or IIB Cervical Cancer: A Systematic Review and Meta-Analysis. Dis. Markers 2020, 2020, 7415056. [CrossRef]

51. Grover, S.; Longo, J.; Einck, J.; Puri, P.; Brown, D.; Chino, J.; Mahantshetty, U.; Yashar, C.; Erickson, B. The Unique Issues With Brachytherapy in Low- and Middle-Income Countries. Glob. Health Disparities 2017, 27, 136-142. [CrossRef]

52. Pfister, C.; Gravis, G.; Fléchon, A.; Soulié, M.; Guy, L.; Laguerre, B.; Mottet, N.; Joly, F.; Allory, Y.; Harter, V.; et al. Randomized Phase III Trial of Dose-Dense Methotrexate, Vinblastine, Doxorubicin, and Cisplatin, or Gemcitabine and Cisplatin as Perioperative Chemotherapy for Patients with Muscle-Invasive Bladder Cancer. Analysis of the GETUG/AFU V05 VESPER Trial Secondary Endpoints: Chemotherapy Toxicity and Pathological Responses. Eur. Urol. 2021, 79, 214-221. [CrossRef] [PubMed]

53. Haddad, R.I.; Posner, M.; Hitt, R.; Cohen, E.E.W.; Schulten, J.; Lefebvre, J.-L.; Vermorken, J.B. Induction Chemotherapy in Locally Advanced Squamous Cell Carcinoma of the Head and Neck: Role, Controversy, and Future Directions. Ann. Oncol. Off. J. Eur. Soc. Med. Oncol. 2018, 29, 1130-1140. [CrossRef] [PubMed]

54. Hsieh, C.-Y.; Lein, M.-Y.; Yang, S.-N.; Wang, Y.-C.; Lin, Y.-J.; Lin, C.-Y.; Hua, C.-H.; Tsai, M.-H.; Lin, C.-C. Dose-Dense TPF Induction Chemotherapy for Locally Advanced Head and Neck Cancer: A Phase II Study. BMC Cancer 2020, 20, 832. [CrossRef]

55. Gupta, S.; Maheshwari, A.; Parab, P.; Mahantshetty, U.; Hawaldar, R.; Sastri Chopra, S.; Kerkar, R.; Engineer, R.; Tongaonkar, H.; Ghosh, J.; et al. Neoadjuvant Chemotherapy Followed by Radical Surgery Versus Concomitant Chemotherapy and Radiotherapy in Patients With Stage IB2, IIA, or IIB Squamous Cervical Cancer: A Randomized Controlled Trial. J. Clin. Oncol. Off. J. Am. Soc. Clin. Oncol. 2018, 36, 1548-1555. [CrossRef] [PubMed]

56. Kenter, G.; Greggi, S.; Vergote, I.; Katsaros, D.; Kobierski, J.; Massuger, L.; van Doorn, H.C.; Landoni, F.; Van Der Velden, J.; Reed, N.S.; et al. Results from Neoadjuvant Chemotherapy Followed by Surgery Compared to Chemoradiation for Stage Ib2-IIb Cervical Cancer, EORTC 55994. J. Clin. Oncol. 2019, 37, 5503. [CrossRef] 